\title{
FUNGAL KERATITIS WITH CORNEAL ULCER IN FARMER
}

\author{
Nugraha Wahyu Cahyana \\ Ophthalmology Departement, Faculty of Medicine, Jember University \\ fk@unej.ac.id
}

\begin{abstract}
Fungal keratitis is important cause of corneal blindness all over the world especially in develoving countries. Fungal Keratitis can diagnosis by slitlamp biomicroscopic examination and culture are essential for early specific diagnosis and must be taken consideratiaon to establish the most effective treatment and avoid severe complications. The study was present a case of Fungal Keratitis in Farmer with corneal ulcer that caused by rice seeds corneal corpus allienum. Corneal ulcer is complication was caused by not effective therapy especially Steroid topical that should be avoid. The culture test result was $C$. Albicans and was treated with natamycin 5\% Eye drops. Finnaly, patients have a good clinical response, however with sequel of decreased visual acuity.
\end{abstract}

Keyword: Corneal Ulcer, farmer, Fungal keratitis , steroid therapy

\section{Introduction}

Fungal Keratitis is an inflammatory disorder of the cornea and its tissues caused by fungi infection [1], and is the most common form of oculomycosis. This ocular disorder is of public concern, as it is detrimental to ocular health, leading to visual impairment and blindness if rapid diagnosis and treatment is not instituted. The combined effect of its cost, protracted treatment and requirement for regular medical visits for follow-ups poses a burden on affected individuals, whose productivity could be affected with the loss of working hours due to residual visual impairment that occurs after treatment.

Fungal Keratitis constitutes between 17 and $60 \%$ of all culture-proven microbial keratitis globally, is relatively common in the tropical regions of the world and has very debilitating effects on the vision of affected individuals [2]. Risk factors commonly implicated in Fungal Keratitis are corneal trauma, contact lens wear, prolonged use of topical or systemic corticosteroids, systemic diseases such as diabetes, previous ocular surgery and ocular surface disease [2].
In developing countries, Fungal Keratitis is historically associated with trauma from vegetative matter or objects contaminated with soil. In developed countries, however, Fungal Keratitis keratomycosis is often associated with contact lens wear, with as many as 60 species of fungi having been found to cause the condition , with filamentous fungi, such as Fusarium species, and yeast-like species, such as Candida, being most implicated [3]. Patients with Fungal Keratitis usually report the sudden onset of pain, photophobia and reduced vision, with clinical features including an inflamed eye (conjunctival injections), corneal ulcers that often lead to opacification, corneal infiltrate with feathery margins, elevated edges, rough texture, graybrown pigmentation, satellite lesions, endothelial plaque and hypopyon [3].

\section{Case report}

A farmer, male 45 -year-old presented with complaints of pain and defective vision in the right eye for three weeks (Figure 1). He gave history of foreign body in the Cornea that caused by rice seeds and eye topical drop by mixture of polidemicin, polimicin and corticosteroid. There was blurred vision, redness, watering, pain and 
photophobia. On examination, the visual acuity was $5 / 60$. Slit lamp evaluation of the right eye revealed a full thickness corneal ulcer. The margins of the ulcer were irregular, base appeared as a sloughing raised mass and the peripheral rim of the cornea was vascularized. Hypopyon filled the lower third of anterior chamber and ocular tension was fairly high. Using standard techniques, corneal scrapings, obtained by a sterile blade no 15 on Bard Parker handle, were inoculated directly onto Sabouraud's media, corn-meal agar, blood agar, and mac conkey agar. These were incubated at room temperature for 4 to $6 \mathrm{wk}$. The growth of fungus was obtained within $8 \mathrm{~d}$ to $14 \mathrm{~d}$ and cultures were discarded after $45 \mathrm{~d}$. The fungi were isolated and identified. The fungus was identified as Candida. The species was confirmed as albicans. The fungus isolated was C. albicans Based on clinical impression, the patient was started on 5\% topical natamycin drops along with $150 \mathrm{mg}$ oral fluconazole twice a day. The patient's have a good clinical response, however with sequel of decreased visual acuity (Figure 2).

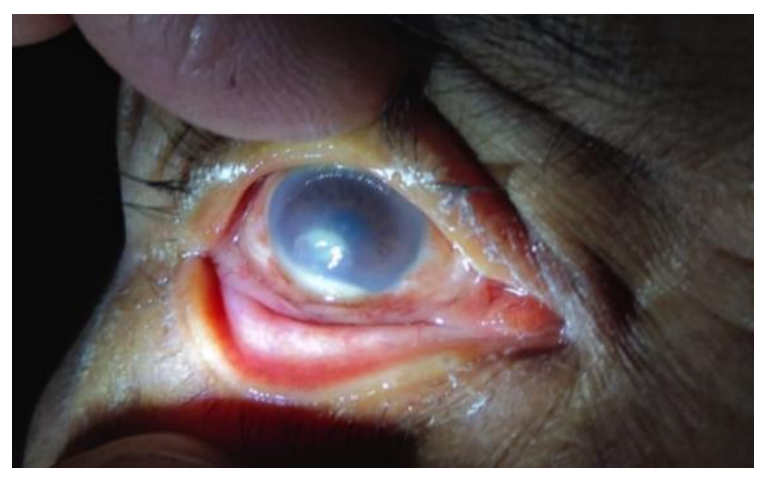

Figure 1. Keratomycosis Candida before treatment

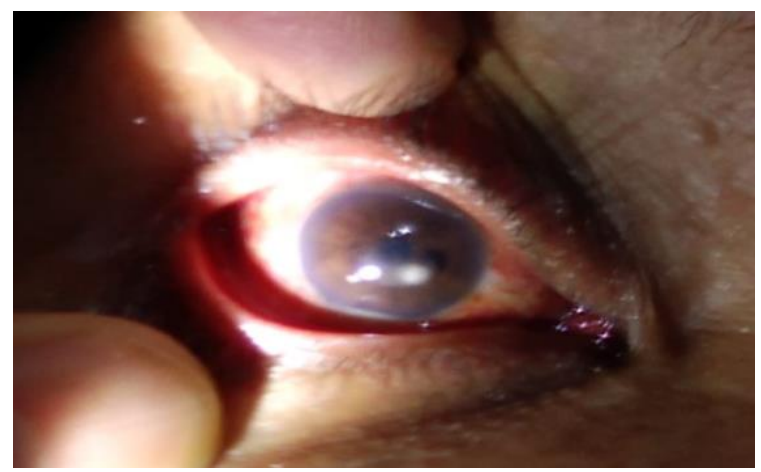

Figure 2. Keratomycosis Candida after treatment

\section{Discussion}

Fungal Keratitis is characterized by stromal infiltration caused by fungi. Fungi are ubiquitous organisms as they are present in environment as a saprotrophs. These fungi cause infection when there is a breach in corneal epithelium as epithelium acts as a barrier to microorganisms, but some of them especially Fusarium spp. may penetrate the intact cornea. World Health Organization reported that corneal diseases are the major cause of monocular blindness after cataract. Around 1.5 to 2 million new cases of corneal ulcer are reported annually [1].

Fungal Keratitis a major cause of monoocular visual disability in developing countries. The first case of Fungal Keratitis was reported by Professor Theodor Leber in 1879 and since then there has been a rapid rise in the diagnosis of fungal corneal ulcer [4]. This is either due to increased awareness among clinicians with better diagnostic facilities or really increasing number of infected cases. Injudicious use of corticosteroid and antibiotics increases the frequency of fungal corneal ulcer. It is estimated that in India, prevalence of fungal corneal ulcer is $44-47 \%$ [5, 6]. compared to $17-36 \%$ worldwide [7]. High prevalence of fungal corneal ulcer is attributed to tropical climate and agriculture as a major occupation. Indians are at a high risk of Fungal Keratitis due to high vulnerability for occupational trauma especially by organic materials and sand particles. Fungi is oportunistic pathogens as they cause tissue invasion only in immuno-compromised state or after direct entry of pathogens in a closed ocular space. The risk factor for the case is traumatic that was caused by rice sheeds corneal trauma. Trauma especially by vegetative material, sand or dust particles and the tail of an animal is the major predisposing factor in $55-65 \%$ cases of fungal corneal ulcer $[5,7]$.

Despite reducing inflammation, topical corticosteroid disability in developing countries. The first case of Fungal Keratitis was reported by Professor Theodor Leber in 1879 and since then there has been a rapid rise in the diagnosis of fungal corneal ulcer [4]. This is either due to increased awareness among clinicians with better diagnostic facilities or really increasing number of infected cases. Injudicious use of

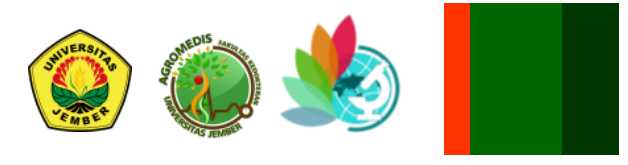


corticosteroid and antibiotics increases the frequency of fungal corneal ulcer. It is estimated that in India, prevalence of fungal corneal ulcer is $44-47 \%$ (Srinivasan instillation enhances fungal growth $[8,9]$. Contact lens users are also at high risk for developing ulcer especially by Fusarium spp. as there are micro epithelial erosions with the use of contact lens $[2,10]$. Other risk factors include corneal surface disorders like dry eyes, corneal dystrophy, exposure keratitis and previous corneal surgery $[6,11]$.

Filamentous fungi are the major pathogen causing fungal keratitis. Hyaline fungi (Aspergillus and Fusarium spp.) have a major preponderance followed by Phaeoid fungi (Curvularia and Bipolaris spp.) [3,12]. Candida spp., a yeast like fungi, have a low preponderance in developing countries. Until recently more than 70 different species of fungi have been identified as the causative agent for fungal corneal ulcer.

Early and prompt diagnosis of fungal corneal ulcer is essential to prevent devastating complications. A good clinical evaluation including relevant clinical history and slit- lamp examination is required to suspect a case of fungal corneal ulcer. The duration of infection in fungal ulcer is usually long. Fungal keratitis can involve any part of cornea. In untreated cases, fungal corneal ulcer is seen to have feathery borders or hyphate edges. Entire lesion or large area of the lesion is elevated well above the surrounding area. Ulcer may be surrounded by a clear rim (devoid of fungi) known as 'Immune Ring of Wessley'. This ring represents immune response against fungi. Non-specific satellite lesions or discrete stromal infiltrates surrounding the ulcer is another common finding. Fungal keratitis, caused by phaeoid fungi has brownblack pigmentation on the surface of corneal ulcer. Hypopyon, the collection of exudates in anterior chamber, is present in around 50\% of cases. Signs of inflammation like pain and ciliary congestion are usually minimal compared to bacterial keratitis. Typical clinical features of fungal corneal ulcer may be masked by instillation of drugs.

Laboratory diagnosis is essential for accurate diagnosis of etiological agents. Prompt and effective treatment, not only slows the progression of the disease, but also results in early healing of the ulcer. False negative diagnosis not only delays the specific antifungal therapy, but also injudicious use of medication leads to rapid growth of organisms. For a better and reliable diagnosis, cornea is scraped several times so that adequate sample material is obtained for microbiological examination.

Using plain $10 \% \mathrm{KOH}$ wet mount, some counter stain substances like India ink, lactophenol cotton blue, ethylene blue and calcofluor white stain can also be used. These substances bind with fungal cell wall component and gives counter stain to the fungal elements resulting in better identification. But they failed to differentiate the types of fungi whether they are hyaline or phaeoid. $\mathrm{KOH}$ wet mount is easy, simple to perform, less time consuming and cost effective. It can also be performed in remote areas, where other facilities of diagnosis are not available. But certain expertise is required to identify and interpret the results. Sensitivity of $\mathrm{KOH}$ varies from 33 to $100 \%$ Lisegang et al., found $33 \%$ sensitivity of $10 \% \mathrm{KOH}$, compared to $100 \%$ sensitivity as reported by S. Sharma in detection of fungal elements in culture proven cases. Sensitivity of $\mathrm{KOH}$ is increased after addition of counterstaining substance like lactophenol cotton blue or fluorescent calcofluor white stain.

Giemsa is a compound stain formed by interaction of methylene blue and eosin. When methylene blue is exposed to acid, alkali or ultraviolet rays, a large number of oxidation products (methylene azure) are formed. In this staining, smear is fixed in methanol and then exposed to Giemsa stain. This stain is used to evaluate the cytology of corneal scrapes, aqueous and vitreous aspirates, pus and necrotic materials. It stains yeast cells and fungal hyphae as purplish blue. Apart from fungi, Acanthamoeba cysts are easily visualized. Sensitivity of Giemsa stain varies from 66- $85 \%$ in culture proven fungal ulcer cases [13]. Drawbacks of this stain are similar to the Gram's stain like artefact, debris and thick smear that interfere with the results.

Culture is considered as gold standard for diagnosis of fungal infection. Culture should be done on freshly prepared Sabouraud's Dextrose Agar (SDA) and Blood Agar. After collection of appropriate sample, it should be inoculated on
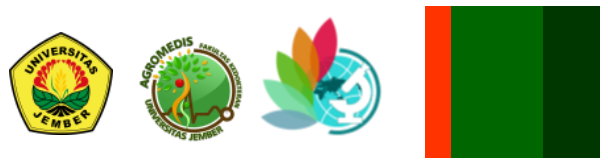
SDA in "C" shape pattern. Growth occurring within the streak lines should be considered as significant as fungi are the commonest laboratory contaminants. Liquid media (BHI) should also be inoculated to enrich the fungal element.

Sabouraud's Dextrose Agar is the most commonly used media for fungal cultivation. Media is made selective by addition of antibacterial antibiotics such as chloramphenicol $(40 \mu \mathrm{m} / \mathrm{ml})$ or gentamicin $(50 \mu \mathrm{m} / \mathrm{ml})$ as these substances inhibit the concomitant bacterial pathogens. Cyclohexamide should not be incorporated in media as it inhibits most of the fungi implicated in ocular fungi like Aspergillus, Penicillium marneffei and Scytalidium spp. Plates are incubated at $25^{\circ} \mathrm{C}$ in BOD incubator.

\section{Conclusion}

Indonesian farmers have highrisk of Fungal keratitis. It was reported in Farmers patiens with corneal ulcer that caused by rice seeds corneal corpus allienum. Corneal ulcer is complication was caused by not effective therapy especially Steroid topical that should be avoid. The patients was treated by natamycin $5 \%$ Eye drops with a good clinical response, however have sequel of decreased visual acuity.

\section{References}

[1] Whitcher JP, Srinivasan M, Upadhyay MP. Corneal blindness: a global perspective. Bull World Health Organ. 2001; 79(3):21421.

[2] Alfonso EC, Cantu-Dibildox J, Munir WM, Miller D, O'Brien TP, Karp CL, Yoo SH, Forster RK, Culbertson WW, Donaldson K, Rodila J, Lee Y. Insurgence of Fusarium keratitis associated with contact lens wear. Arch Ophthalmol. 2006; 124(7):941-7.

[3] Basak SK, Basak S, Mohanta A, Bhowmick A. Epidemiological and microbiological diagnosis of suppurative keratitis in gangetic West Bengal, Eastern India. Indian J Ophthalmol 2005; 53:17-22.

[4] Anderson B. Jr, Chick EW. Mycokeratitis: Treatment of fungal corneal ulcers with amphotericin B and mechanical debridement. South Med J. 1963; 56:270-4.
[5] Srinivasan M. Fungal keratitis. Curr Opin Ophthalmol 2004; 15:321-327.

[6] Thomas PA. Mycotic keratitis-an underestimated mycosis. J Med Vet Mycol.1994; 32:235-256.

[7] Upadhyay MP, Karmacharya PC, Koirala S, Tuladhar NR, Bryan LE, Smolin G, Whitcher JP. Epidemiologic characteristic, predisposing factors and etiological diagnosis of corneal ulceration in Nepal Am. J. Ophthalmol. 1991:111 (1): 92-99.

[8] Agarwal LP, Malik SR, Mohan M, Khosla PK. Mycotic Corneal Ulcers. Br. J. Ophthalmol. 1963; 47:109-15.

[9] Forster RK, Rebell G. The diagnosis and management of keratomycoses. I. Cause and diagnosis. Arch Ophthalmol. 1975; 93(10):975-8.

[10] Choi DM, Goldstein MH, Salierno A, Driebe WT. Fungal keratitis in a daily disposable soft contact lens wearer. CLAO J. 2001; 27(2):111-2.

[11] Anderson B, Roberts SS Jr, Gonzalez C, Chick EW. Mycotic ulcerative keratitis. AMA Arch Ophthalmol. 1959; 62(2):16997.

[12] Vijaya D, Sumathi, Malini. Keratomycosis due to Fusarium oxysporum-A case report. Indian J. Pathol. Microbiol. 2001; 44:337-8.

[13] Gopinathan U, Garg P, Fernandes M, Sharma S, Athmanathan S, Rao GN. The epidemiological features and laboratory results of fungal keratitis: a 10-year review at a referral eye care center in South India. Cornea. 2002; 21:555-559. 